\title{
MODIFIED THREE STEP ITERATIVE PROCESS WITH ERRORS FOR COMMON FIXED POINT OF GENERALIZED ASYMPTOTICALLY QUASI-NONEXPANSIVE MAPPINGS
}

\author{
SEYIT TEMIR AND HUKMI KIZILTUNC
}

\begin{abstract}
In this paper we introduce to modified three step iterative process with errors for approximating the common fixed point for generalized asymptotically quasinonexpansive mappings and prove some strong convergence results for the iterative sequences iterations with errors in real Banach spaces. The results obtained in this paper extend and improve the recent ones announced by Lan [4], Nantadilok [10], Saluja and Nashine [14] and Yang et all. [18] and many others.
\end{abstract}

\section{Introduction}

The iterative approximation problems for nonexpansive mapping, asymptotically nonexpansive mapping and asymptotically quasi-nonexpansive mapping were studied Ghosh and Debnath [1], Goebel and Kirk [3], Liu [5, 6], Petryshyn and Williamson [13] in the settings of Hilbert spaces and uniformly convex Banach spaces. The class of asymptotically nonexpansive maps which an important generalization of the class nonexpansive maps was introduced by Goebel and Kirk [3]. They proved that every asymptotically nonexpansive self-mapping of a nonempty closed convex bounded subset of a uniformly convex Banach space has a fixed point. Also in [3], they extended this result to broader class of uniformly Lipschitzian mappings. Since 1972, iterative techniques for convergence to fixed points of nonexpansive mappings and their generalizations in setting of Hilbert spaces or Banach spaces have been studied by many authors. For example, in 1973, Petryshyn and Williamson [13] proved a necessary and sufficient condition for a Mann iterative sequence to convergence to fixed points for quasi-nonexpansive mappings. In 1997, Ghosh and Debnath [1] extended Petryshyn and Williamson's results and gave some necessary and sufficient conditions for Ishikawa iterative sequence to converge to fixed points for quasi-nonexpansive mappings. Subsequently, in 2001 and 2002, Liu Qihou [5, 6] extended the results of Ghosh and Debnath to the more

Corresponding author: Seyit Temir.

2010 Mathematics Subject Classification. 47H09, 47H10.

Key words and phrases. Asymptotically quasi-nonexpansive mappings, modified three-step iterative sequence with errors, common fixed points, convergence theorems. 
general asymptotically quasi-nonexpansive mapping and gave some necessary and sufficient conditions for Ishikawa iterative sequence and Ishikawa iterative sequence with errors to converge to fixed points for asymptotically quasi-nonexpansive mappings in Banach spaces and uniformly Banach spaces. Recently, in 2006, Lan [4] introduced a new class of iterative processes with errors for approximating the common fixed point of two generalized asymptotically quasi-nonexpansive mappings and gave some strong convergence results for Ishikawa iterative sequence to fixed point for this class of mappings.

Noor [12] introduced a three-step iterative scheme and studied the approximate solutions of variational inclusion in Hilbert spaces. Glowinski and Le Tallec [2] applied a three step iterative process for finding the approximate solutions of liquid crystal theory, and eigenvalue computation. It has been shown in [2] that the three-step iterative scheme gives better numerical results than the two-step and one-step approximate iterations. $\mathrm{Xu}$ and Noor [17] introduced and studied a three-step scheme to approximate fixed point of asymptotically nonexpansive mappings in a Banach space. Nilsrakoo and Saejung [11] defined a new three-step iterations which is an extension of Noor iterations and gave some weak and strong convergence theorems of the modified Noor iterations for asymptotically nonexpansive mappings in Banach space. It is clear that the modified Noor iterations include Mann iterations [7], Ishikawa iterations [8] and original Noor iterations [12] as special cases. In 2007 , Nammanee and Suantai [9] gave convergence criteria of modified three-step iterations with errors for asymptotically nonexpansive mappings.Very recently, Nantadilok [10], introduced three step iteration schemes with errors for approximating the common fixed point of three generalized asymptotically quasi-nonexpansive mappings. More recently, Saluja and Nashine [14] extended and improved the result of Nantadilok [10] and Lan [4] and obtained a convergence result of three step iteration schemes with errors for three generalized asymptotically quasinonexpansive mappings .

The aim of this paper is to obtain convergence result of modified three iterative process with errors for generalized asymptotically quasi-nonexpansive mappings in real Banach spaces. Thus, our results will improve and generalize corresponding results of Lan [4], Nantadilok [10], Saluja and Nashine [14] and Yang et al [18].

\section{Preliminaries and notations}

Throughout this paper, we assume that $X$ be a real Banach space, $K$ is a nonempty closed convex subset $X$ and $F(T)$ is the set of fixed points of mapping $T$.

Definition 2.1. (1) A mapping $T$ is called nonexpansive if

$$
\|T x-T y\| \leq\|x-y\|
$$

for all $x, y \in K$. 
(2) $T$ is called quasi-nonexpansive if $F(T) \neq \varnothing$ and

$$
\|T x-p\| \leq\|x-p\|
$$

for all $x \in K$ and $p \in F(T)$.

(3) $T$ is called asymptotically nonexpansive mapping if there exist a sequence $\left\{r_{n}\right\} \subset[0, \infty)$ with $\lim _{n \rightarrow \infty} r_{n}=0$ such that

$$
\left\|T^{n} x-T^{n} y\right\| \leq\left(1+r_{n}\right)\|x-y\|
$$

for all $x, y \in K$ and $n \geq 1$.

(4) $T$ is called asymptotically quasi-nonexpansive mapping if $F(T) \neq \varnothing$ and there exist a sequence $\left\{r_{n}\right\} \subset[0, \infty)$ with $\lim _{n \rightarrow \infty} r_{n}=0$ such that

$$
\left\|T^{n} x-p\right\| \leq\left(1+r_{n}\right)\|x-p\|
$$

for all $x \in K, p \in F(T)$ and $n \geq 1$.

(5) $T$ is called generalized asymptotically quasi-nonexpansive mapping with respect to $r_{n}$ and $s_{n}$ if there exist $\left\{r_{n}\right\}$ and $\left\{s_{n}\right\} \subset[0,1)$ with $\lim _{n \rightarrow \infty} r_{n}=0$ and $\lim _{n \rightarrow \infty} s_{n}=0$ such that

$$
\left\|T^{n} x-p\right\| \leq\left(1+r_{n}\right)\|x-p\|+s_{n}\left\|x-T^{n} x\right\|
$$

for all $x \in K, p \in F(T)$ and $n \geq 1$.

It is clear that if $F(T)$ is nonempty, then the asymptotically nonexpansive mapping, the asymptotically quasi-nonexpansive mapping, and the generalized quasi-nonexpansive mapping are all the generalized asymptotically quasi-nonexpansive mapping.

Lemma 2.1 ([16]). Let $\left\{\alpha_{n}\right\},\left\{t_{n}\right\}$ and $\left\{\sigma_{n}\right\}$ be sequences of nonnegative real sequences satisfying the following conditions: $\forall n \geq 1, \alpha_{n+1} \leq\left(1+\sigma_{n}\right) \alpha_{n}+t_{n}$, where $\sum_{n=0}^{\infty} \sigma_{n}<\infty$ and $\sum_{n=0}^{\infty} t_{n}<\infty$. Then $\lim _{n \rightarrow \infty} \alpha_{n}$ exists.

Let $X$ be a real Banach space, $K$ be a nonempty closed convex subset of $X$. Let $T_{i}: K \rightarrow K$ $(i=1,2,3)$ be given generalized asymptotically quasi-nonexpansive mappings. Then for a given $x_{1} \in K$ and $n \geq 1$, compute the iterative sequences $\left\{z_{n}\right\},\left\{y_{n}\right\},\left\{x_{n}\right\}$ defined by

$$
\left\{\begin{array}{l}
z_{n}=\left(1-a_{n 3}-\mu_{n 3}\right) x_{n}+a_{n 3} T_{3}^{n} x_{n}+\mu_{n 3} u_{n 3} \\
y_{n}=\left(1-a_{n 2}-b_{n 2}-\mu_{n 2}\right) x_{n}+a_{n 2} T_{2}^{n} z_{n}+b_{n 2} T_{2}^{n} x_{n}+\mu_{n 2} u_{n 2} \\
x_{n+1}=\left(1-a_{n 1}-b_{n 1}-c_{n 1}-\mu_{n 1}\right) x_{n}+a_{n 1} T_{1}^{n} y_{n}+b_{n 1} T_{1}^{n} z_{n}+c_{n 1} T_{1}^{n} x_{n}+\mu_{n 1} u_{n 1}, \forall n \geq 1,
\end{array}\right.
$$

where for $i=1,2,3, \quad\left\{a_{n i}\right\},\left\{\mu_{n i}\right\},\left\{b_{n 2}\right\},\left\{b_{n 1}\right\}, \quad\left\{c_{n 1}\right\},\left\{a_{n 3}+\mu_{n 3}\right\},\left\{a_{n 2}+b_{n 2}+\mu_{n 2}\right\},\left\{a_{n 1}+\right.$ $\left.b_{n 1}+c_{n 1}+\mu_{n 1}\right\}$ are appropriate sequences in $[0,1]$ and $\left\{u_{n i}\right\}$ are bounded sequences in $K$. 
The iterative schemes (2.1) are called the modified three-step iterations with errors [18]. If $T_{1}=T_{2}=T_{3}=T$, and $c_{n 1}=0$, then (2.1) reduces to the modified Noor iterations defined in Nammanee and Suantai [9]. If $T_{1}=T_{2}=T_{3}=T, \mu_{n i}=0 \quad(i=1,2,3)$ and $c_{n 1}=0$, then (2.1) reduces to the modified Noor iterations defined in Suantai [15]. If $T_{1}=T_{2}=T_{3}=T, \mu_{n i}=0$ $(i=1,2,3)$ and $b_{n 2}=b_{n 1}=c_{n 1}=0$, then (2.1) reduces to Noor iterations defined by Xu and Noor [17]. If $b_{n 2}=b_{n 1}=c_{n 1}=0$, then (2.1) reduces to three-step iterations defined by Saluja and Nashine[14]. Nantadilok[10] was defined three-step iterations as follows:

$$
\left\{\begin{array}{l}
z_{n}=c_{n} T_{3}^{n} x_{n}+\left(1-c_{n}\right) x_{n}+\mu_{n 3} u_{n 3} \\
y_{n}=b_{n} T_{2}^{n} z_{n}+\left(1-b_{n}\right) x_{n}+\mu_{n 2} u_{n 2} \\
x_{n+1}=a_{n} T_{1}^{n} y_{n}+\left(1-a_{n}\right) x_{n}+\mu_{n 1} u_{n 1}, \forall n \geq 1,
\end{array}\right.
$$

where for $i=1,2,3, \quad \mu_{n i}, \quad\left\{a_{n}\right\},\left\{b_{n}\right\},\left\{c_{n}\right\}$ are sequences in [0,1] satisfying some conditions.

If $\left\{c_{n}\right\}=\left\{\mu_{n 3}\right\}=0$, then (2.2) reduces to generalized modified Ishikawa iterations[4] as follows:

$$
\left\{\begin{array}{l}
y_{n}=b_{n} T^{n} x_{n}+\left(1-b_{n}\right) x_{n}+\mu_{n 2} u_{n 2} \\
x_{n+1}=a_{n} T^{n} y_{n}+\left(1-a_{n}\right) x_{n}+\mu_{n 1} u_{n 1}, \forall n \geq 1,
\end{array}\right.
$$

where for $i=1,2, \mu_{n i},\left\{a_{n}\right\}, \quad\left\{b_{n}\right\}$ are sequences in $[0,1]$ satisfying some conditions. If $\left\{b_{n}\right\}=$ $\left\{\mu_{n 2}\right\}=0$, then (2.3) reduces to general modified Mann iterative process as follows:

$$
x_{n+1}=a_{n} T^{n} x_{n}+\left(1-a_{n}\right) x_{n}+\mu_{n 1} u_{n 1}, \quad \forall n \geq 1,
$$

where $\mu_{n 1}$ and $\left\{a_{n}\right\}$ are sequences in $[0,1]$ satisfying some conditions.

\section{Main results}

In this section, we prove some strong convergence theorems of the modified three step iterative sequence given in (2.1) to common fixed point for generalized asymptotically quasinonexpansive mappings in real Banach spaces.

Theorem 3.1. Let $X$ be a real Banach space and $K$ be a nonempty closed convex subset of $X$ and for $i=1,2,3, \quad T_{i}: K \rightarrow K$ be generalized asymptotically quasi-nonexpansive mappings with respect to $\left\{r_{n i}\right\}$ and $\left\{s_{n i}\right\} \subset[0,1)$ such that $\mathscr{F}=\bigcap_{i=1}^{3} F\left(T_{i}\right) \neq \varnothing$ in $K$, and $\sum_{n=1}^{\infty} \frac{2 s_{n}+r_{n}}{1-s_{n}}<\infty$ where $r_{n}=\max \left\{r_{n 1}, r_{n 2}, r_{n 3}\right\}, s_{n}=\max \left\{s_{n 1}, s_{n 2}, s_{n 3}\right\}$ with $\lim _{n \rightarrow \infty} r_{n}=0$ and $\lim _{n \rightarrow \infty} s_{n}=0$. Assume that $\sum_{n=1}^{\infty} \mu_{n 1}<\infty, \quad \sum_{n=1}^{\infty} \mu_{n 2}<\infty, \sum_{n=1}^{\infty} \mu_{n 3}<\infty$. Then the iterative sequence $\left\{x_{n}\right\}$ defined by (2.1) converges strongly a common fixed point $p$ of $T_{1}, T_{2}, T_{3}$ if and only if $\lim _{n \rightarrow \infty} d\left(x_{n}, p\right)=0$.

Proof. The necessity is obvious and so it is omitted. 
Now, we prove the sufficiency. Let $p \in \bigcap_{i=1}^{3} F\left(T_{i}\right)$, then it follows from (5), we have

$$
\begin{aligned}
\left\|x_{n}-T_{3}^{n} x_{n}\right\| & \leq\left\|x_{n}-p\right\|+\left\|T_{3}^{n} x_{n}-p\right\| \\
& \leq\left\|x_{n}-p\right\|+\left(1+r_{n 3}\right)\left\|x_{n}-p\right\|+s_{n 3}\left\|x_{n}-T_{3}^{n} x_{n}\right\| \\
& \leq\left(2+r_{n 3}\right)\left\|x_{n}-p\right\|+s_{n 3}\left\|x_{n}-T_{3}^{n} x_{n}\right\| \\
& \leq\left(2+r_{n}\right)\left\|x_{n}-p\right\|+s_{n}\left\|x_{n}-T_{3}^{n} x_{n}\right\|
\end{aligned}
$$

which implies that

$$
\left\|x_{n}-T_{3}^{n} x_{n}\right\| \leq \frac{2+r_{n}}{1-s_{n}}\left\|x_{n}-p\right\|
$$

Similarly, we have

$$
\begin{aligned}
\left\|x_{n}-T_{2}^{n} x_{n}\right\| & \leq \frac{2+r_{n}}{1-s_{n}}\left\|x_{n}-p\right\| . \\
\left\|x_{n}-T_{1}^{n} x_{n}\right\| & \leq \frac{2+r_{n}}{1-s_{n}}\left\|x_{n}-p\right\| . \\
\left\|z_{n}-T_{2}^{n} z_{n}\right\| & \leq \frac{2+r_{n}}{1-s_{n}}\left\|z_{n}-p\right\| . \\
\left\|z_{n}-T_{1}^{n} z_{n}\right\| & \leq \frac{2+r_{n}}{1-s_{n}}\left\|z_{n}-p\right\| . \\
\left\|y_{n}-T_{1}^{n} y_{n}\right\| & \leq \frac{2+r_{n}}{1-s_{n}}\left\|y_{n}-p\right\| .
\end{aligned}
$$

Since for $i=1,2,3,\left\{u_{n i}\right\}$ are bounded sequences in $K$, for any given $p \in \mathscr{F}$, we can set $M_{i}=$ $\sup \left\{\left\|u_{n i}-p\right\|: n \geq 1\right\},(i=1,2,3), \quad M=\max \left\{M_{i}: i=1,2,3\right\}$.

It follows from (2.1), (3.1) and (5) that

$$
\begin{aligned}
\left\|z_{n}-p\right\| & =\left\|\left(1-a_{n 3}-\mu_{n 3}\right) x_{n}+a_{n 3} T_{3}^{n} x_{n}+\mu_{n 3} u_{n 3}-p\right\| \\
& \leq\left(1-a_{n 3}-\mu_{n 3}\right)\left\|x_{n}-p\right\|+a_{n 3}\left\|T_{3}^{n} x_{n}-p\right\|+\mu_{n 3}\left\|u_{n 3}-p\right\| \\
& \leq\left(1-a_{n 3}-\mu_{n 3}\right)\left\|x_{n}-p\right\|+a_{n 3}\left(\left(1+r_{n 3}\right)\left\|x_{n}-p\right\|+s_{n 3}\left\|x_{n}-T_{3}^{n} x_{n}\right\|\right)+\mu_{n 3}\left\|u_{n 3}-p\right\| \\
& \leq\left(1-a_{n 3}-\mu_{n 3}\right)\left\|x_{n}-p\right\|+a_{n 3}\left(\left(1+r_{n}\right)\left\|x_{n}-p\right\|+s_{n}\left\|x_{n}-T_{3}^{n} x_{n}\right\|\right)+\mu_{n 3} M_{3} \\
& \leq\left(1-a_{n 3}-\mu_{n 3}\right)\left\|x_{n}-p\right\|+a_{n 3}\left(\left(1+r_{n}\right)\left\|x_{n}-p\right\|+s_{n}\left(\frac{2+r_{n}}{1-s_{n}}\right)\left\|x_{n}-p\right\|\right)+\mu_{n 3} M \\
& \leq\left(1-a_{n 3}-\mu_{n 3}\right)\left\|x_{n}-p\right\|+a_{n 3}\left(\frac{1+s_{n}+r_{n}}{1-s_{n}}\right)\left\|x_{n}-p\right\|+\mu_{n 3} M \\
& \leq\left(1+a_{n 3}\left(\frac{1+s_{n}+r_{n}}{1-s_{n}}-1\right)-\mu_{n 3}\right)\left\|x_{n}-p\right\|+\mu_{n 3} M \\
& \leq\left(\frac{1+s_{n}+r_{n}}{1-s_{n}}\right)\left\|x_{n}-p\right\|+\mu_{n 3} M \\
& \leq k_{n}\left\|x_{n}-p\right\|+\mu_{n 3} M
\end{aligned}
$$


where $k_{n}=\frac{1+s_{n}+r_{n}}{1-s_{n}} \rightarrow 1$, since $r_{n} \rightarrow 0$ and $s_{n} \rightarrow 0$ as $n \rightarrow \infty$

which implies that

$$
\left\|z_{n}-p\right\| \leq k_{n}\left\|x_{n}-p\right\|+\mu_{n 3} M .
$$

Again from (2.1), (3.2), (3.4), (5) and (3.7), we have

$$
\begin{aligned}
\left\|y_{n}-p\right\|= & \left\|\left(1-a_{n 2}-b_{n 2}-\mu_{n 2}\right) x_{n}+a_{n 2} T_{2}^{n} z_{n}+b_{n 2} T_{2}^{n} x_{n}+\mu_{n 2} u_{n 2}-p\right\| \\
\leq & \left(1-a_{n 2}-b_{n 2}-\mu_{n 2}\right)\left\|x_{n}-p\right\|+a_{n 2}\left\|T_{2}^{n} z_{n}-p\right\|+b_{n 2}\left\|T_{2}^{n} x_{n}-p\right\|+\mu_{n 2}\left\|u_{n 2}-p\right\| \\
\leq & \left(1-a_{n 2}-b_{n 2}-\mu_{n 2}\right)\left\|x_{n}-p\right\|+a_{n 2}\left(\left(1+r_{n 2}\right)\left\|z_{n}-p\right\|+s_{n 2}\left\|z_{n}-T_{2}^{n} z_{n}\right\|\right) \\
& +b_{n 2}\left(\left(1+r_{n 2}\right)\left\|x_{n}-p\right\|+s_{n 2}\left\|x_{n}-T_{2}^{n} x_{n}\right\|\right)+\mu_{n 2} M_{2} \\
\leq & \left(1-a_{n 2}-b_{n 2}-\mu_{n 2}\right)\left\|x_{n}-p\right\|+a_{n 2}\left(\left(1+r_{n}\right)\left\|z_{n}-p\right\|+s_{n}\left\|z_{n}-T_{2}^{n} z_{n}\right\|\right) \\
& +b_{n 2}\left(\left(1+r_{n}\right)\left\|x_{n}-p\right\|+s_{n}\left\|x_{n}-T_{2}^{n} x_{n}\right\|\right)+\mu_{n 2} M \\
\leq & \left(1-a_{n 2}-b_{n 2}-\mu_{n 2}\right)\left\|x_{n}-p\right\|+a_{n 2}\left(\left(1+r_{n}\right)\left\|z_{n}-p\right\|+s_{n}\left(\frac{2+r_{n}}{1-s_{n}}\right)\left\|z_{n}-p\right\|\right) \\
& +b_{n 2}\left(\left(1+r_{n}\right)\left\|x_{n}-p\right\|+s_{n}\left(\frac{2+r_{n}}{1-s_{n}}\right)\left\|x_{n}-p\right\|\right)+\mu_{n 2} M \\
\leq & \left(1-a_{n 2}-b_{n 2}-\mu_{n 2}\right)\left\|x_{n}-p\right\|+a_{n 2}\left(\frac{1+s_{n}+r_{n}}{1-s_{n}}\right)\left\|z_{n}-p\right\| \\
& +b_{n 2}\left(\frac{1+s_{n}+r_{n}}{1-s_{n}}\right)\left\|x_{n}-p\right\|+\mu_{n 2} M \\
\leq & \left(1-a_{n 2}-b_{n 2}-\mu_{n 2}\right)\left\|x_{n}-p\right\| \\
& +a_{n 2}\left[\left(\frac{1+s_{n}+r_{n}}{1-s_{n}}\right)\left\|x_{n}-p\right\|+\left(\frac{1+s_{n}+r_{n}}{1-s_{n}}\right) \mu_{n 3} M\right] \\
& +b_{n 2}\left(\frac{1+s_{n}+r_{n}}{1-s_{n}}\right)\left\|x_{n}-p\right\|+\mu_{n 2} M \\
\leq & {\left.\left[1+a_{n 2}\left(\left(\frac{1+s_{n}+r_{n}}{1-s_{n}}\right)\right)^{2}-1\right)-\mu_{n 2}\right]\left\|x_{n}-p\right\| } \\
& +b_{n 2}\left[\left(\frac{1+s_{n}+r_{n}}{1-s_{n}}\right)-1\right]\left\|x_{n}-p\right\|+a_{n 2}\left(\frac{1+s_{n}+r_{n}}{1-s_{n}}\right) \mu_{n 3} M+\mu_{n 2} M \\
\leq & {\left[1+a_{n 2}\left(\left(k_{n}\right)^{2}-1\right)\right]\left\|x_{n}-p\right\| } \\
\leq & {\left[1+\left(k_{n 2}^{2}\left[\left(k_{n}\right)-1\right]\left\|x_{n}-p\right\|+a_{n 2}\left(k_{n}\right) \mu_{n 3} M+\mu_{n 2} M\right.\right.} \\
& \\
&
\end{aligned}
$$

where $k_{n}=\frac{1+s_{n}+r_{n}}{1-s_{n}} \rightarrow 1$, since $r_{n} \rightarrow 0$ and $s_{n} \rightarrow 0$ as $n \rightarrow \infty$

which implies that

$$
\left\|y_{n}-p\right\| \leq\left(1+\left(k_{n}^{2}-1\right)+\left(k_{n}-1\right)\right)\left\|x_{n}-p\right\|+\left(\mu_{n 3}+\mu_{n 2}\right) M^{1},
$$


where $M^{1}=\sup _{n \geq 1} k_{n} \cdot M$

Again from (2.1), (5), (3.3), (3.5), (3.6), (3.7) and (3.8) we have

$$
\begin{aligned}
\left\|x_{n+1}-p\right\|= & \left\|\left(1-a_{n 1}-b_{n 1}-c_{n 1}-\mu_{n 1}\right) x_{n}+a_{n 1} T_{1}^{n} y_{n}+b_{n 1} T_{1}^{n} z_{n}+c_{n 1} T_{1}^{n} x_{n}+\mu_{n 1} u_{n 1}-p\right\| \\
\leq & \left(1-a_{n 1}-b_{n 1}-c_{n 1}-\mu_{n 1}\right)\left\|x_{n}-p\right\|+a_{n 1}\left\|T_{1}^{n} y_{n}-p\right\|+b_{n 1}\left\|T_{1}^{n} z_{n}-p\right\| \\
& +c_{n 1}\left\|T_{1}^{n} x_{n}-p\right\|+\mu_{n 1}\left\|u_{n 1}-p\right\| \\
\leq & \left(1-a_{n 1}-b_{n 1}-c_{n 1}-\mu_{n 1}\right)\left\|x_{n}-p\right\|+a_{n 1}\left(\left(1+r_{n 1}\right)\left\|y_{n}-p\right\|+s_{n 1}\left\|y_{n}-T_{1}^{n} y_{n}\right\|\right) \\
& +b_{n 1}\left(\left(1+r_{n 1}\right)\left\|z_{n}-p\right\|+s_{n 1}\left\|z_{n}-T_{1}^{n} z_{n}\right\|\right) \\
& +c_{n 1}\left(\left(1+r_{n 1}\right)\left\|x_{n}-p\right\|+s_{n 1}\left\|x_{n}-T_{1}^{n} x_{n}\right\|\right)+\mu_{n 1} M_{1}
\end{aligned}
$$

$\left\|x_{n+1}-p\right\| \leq\left(1-a_{n 1}-b_{n 1}-c_{n 1}-\mu_{n 1}\right)\left\|x_{n}-p\right\|+a_{n 1}\left(\left(1+r_{n}\right)\left\|y_{n}-p\right\|+s_{n}\left\|y_{n}-T_{1}^{n} y_{n}\right\|\right)$

$$
\begin{aligned}
& +b_{n 1}\left(\left(1+r_{n}\right)\left\|z_{n}-p\right\|+s_{n}\left\|z_{n}-T_{1}^{n} z_{n}\right\|\right) \\
& +c_{n 1}\left(\left(1+r_{n}\right)\left\|x_{n}-p\right\|+s_{n}\left\|x_{n}-T_{1}^{n} x_{n}\right\|\right)+\mu_{n 1} M \\
\leq & \left(1-a_{n 1}-b_{n 1}-c_{n 1}-\mu_{n 1}\right)\left\|x_{n}-p\right\|+a_{n 1}\left(\left(1+r_{n}\right)\left\|y_{n}-p\right\|+s_{n}\left(\frac{2+r_{n}}{1-s_{n}}\right)\left\|y_{n}-p\right\|\right) \\
& +b_{n 1}\left(\left(1+r_{n}\right)\left\|z_{n}-p\right\|+s_{n}\left(\frac{2+r_{n}}{1-s_{n}}\right)\left\|z_{n}-p\right\|\right) \\
& +c_{n 1}\left(\left(1+r_{n}\right)\left\|x_{n}-p\right\|+s_{n}\left(\frac{2+r_{n}}{1-s_{n}}\right)\left\|x_{n}-p\right\|\right)+\mu_{n 1} M \\
\leq & \left(1-a_{n 1}-b_{n 1}-c_{n 1}-\mu_{n 1}\right)\left\|x_{n}-p\right\|+a_{n 1}\left(\frac{1+s_{n}+r_{n}}{1-s_{n}}\right)\left\|y_{n}-p\right\| \\
& +b_{n 1}\left(\frac{1+s_{n}+r_{n}}{1-s_{n}}\right)\left\|z_{n}-p\right\|+c_{n 1}\left(\frac{1+s_{n}+r_{n}}{1-s_{n}}\right)\left\|x_{n}-p\right\|+\mu_{n 1} M \\
\leq & \left(1-a_{n 1}-b_{n 1}-c_{n 1}-\mu_{n 1}\right)\left\|x_{n}-p\right\| \\
& +a_{n 1} k_{n}\left[1+\left(k_{n}^{2}-1\right)+\left(k_{n}-1\right)\left\|x_{n}-p\right\|+\left(\mu_{n 3}+\mu_{n 2}\right) M^{1}\right] \\
& +b_{n 1} k_{n}\left[\left(k_{n}\right)\left\|x_{n}-p\right\|+\mu_{n 3} M\right]+c_{n 1} k_{n}\left\|x_{n}-p\right\|+\mu_{n 1} M
\end{aligned}
$$

$\left\|x_{n+1}-p\right\| \leq\left[1+a_{n 1}\left(k_{n}-1\right)+a_{n 1} k_{n}\left(k_{n}^{2}-1\right)\right.$

$$
\begin{aligned}
& \left.+a_{n 1} k_{n}\left(k_{n}-1\right)+b_{n 1}\left(k_{n}^{2}-1\right)+c_{n 1}\left(k_{n}-1\right)\right]\left\|x_{n}-p\right\| \\
& +a_{n 1} k_{n}\left(\mu_{n 3}+\mu_{n 2}\right) M^{1}+b_{n 1} k_{n} \mu_{n 3} M+\mu_{n 1} M \\
\leq & {\left[1+\left(k_{n}-1\right)+k_{n}\left(k_{n}^{2}-1\right)+k_{n}\left(k_{n}-1\right)+\left(k_{n}^{2}-1\right)+\left(k_{n}-1\right)\right]\left\|x_{n}-p\right\| } \\
& +k_{n}\left(\mu_{n 3}+\mu_{n 2}\right) M^{1}+k_{n} \mu_{n 3} M+\mu_{n 1} M \\
\leq & {\left[1+\left(k_{n}-1\right)+k_{n}\left(k_{n}^{2}-1\right)+k_{n}\left(k_{n}-1\right)+\left(k_{n}^{2}-1\right)+\left(k_{n}-1\right)\right]\left\|x_{n}-p\right\| } \\
& +k_{n}\left(2 \mu_{n 3}+\mu_{n 2}+\mu_{n 1}\right) M^{1}
\end{aligned}
$$

where $k_{n}=\frac{1+s_{n}+r_{n}}{1-s_{n}} \rightarrow 1$, since $r_{n} \rightarrow 0$ and $s_{n} \rightarrow 0$ as $n \rightarrow \infty$ 
which implies that

$$
\begin{aligned}
\left\|x_{n+1}-p\right\| \leq & {\left[1+\left(k_{n}-1\right)+k_{n}\left(k_{n}^{2}-1\right)+k_{n}\left(k_{n}-1\right)+\left(k_{n}^{2}-1\right)+\left(k_{n}-1\right)\right]\left\|x_{n}-p\right\| } \\
& +\left(2 \mu_{n 3}+\mu_{n 2}+\mu_{n 1}\right) M^{2},
\end{aligned}
$$

where $M^{2}=\sup _{n \geq 1} k_{n} \cdot M^{1}$.

Consequently, we can rewrite (3.9) as follows:

$$
\left\|x_{n+1}-p\right\| \leq\left(1+\sigma_{n}\right)\left\|x_{n}-p\right\|+\tau_{n},
$$

where $\sigma_{n}=\left(k_{n}-1\right)+k_{n}\left(k_{n}^{2}-1\right)+k_{n}\left(k_{n}-1\right)+\left(k_{n}^{2}-1\right)+\left(k_{n}-1\right), \tau_{n}=\left(2 \mu_{n 3}+\mu_{n 2}+\mu_{n 1}\right) M^{2}$.

Since $\left\{k_{n}\right\}$ is a nonincreasing bounded sequence, $k_{n}-1=\left(\frac{1+s_{n}+r_{n}}{1-s_{n}}\right)-1=\left(\frac{2 s_{n}+r_{n}}{1-s_{n}}\right)$, the assumption $\sum_{n=1}^{\infty} \frac{2 s_{n}+r_{n}}{1-s_{n}}<\infty, \sum_{n=1}^{\infty}\left(k_{n}-1\right)<\infty$ implies that $\sum_{n=1}^{\infty}\left(k_{n}^{2}-1\right)<\infty$. Therefore, by assumption and $\sum_{n=1}^{\infty} \mu_{n 1}<\infty, \quad \sum_{n=1}^{\infty} \mu_{n 2}<\infty, \quad \sum_{n=1}^{\infty} \mu_{n 3}<\infty$, it follows that $\sum_{n=1}^{\infty} \sigma_{n}<\infty$ and $\sum_{n=1}^{\infty} \tau_{n}<\infty$.

It is well known that in fact, $1+x \leq e^{x}$ for all $x>0$, from (3.10) for all $p \in \mathscr{F}$, we get

$$
\begin{aligned}
\left\|x_{n+m}-p\right\| & \leq\left(1+\sigma_{n+m-1}\right)\left\|x_{n+m-1}-p\right\|+\tau_{n+m-1} \\
& \leq \exp \left(\sigma_{n+m-1}\right)\left(\left\|x_{n+m-1}-p\right\|+\tau_{n+m-1}\right) \\
& \leq \exp \left(\sigma_{n+m-1}+\sigma_{n+m-2}\right)\left(\left\|x_{n+m-2}-p\right\|+\tau_{n+m-2}+\tau_{n+m-1}\right) \\
& \vdots \\
& \leq \exp \left(\sum_{1=n}^{n+m-1} \sigma_{i}\right)\left\|x_{n}-p\right\|+\exp \left(\sum_{1=n}^{n+m-1} \sigma_{i}\right) \sum_{1=n}^{n+m-1} \tau_{i} \\
& \leq R\left\|x_{n}-p\right\|+R \sum_{1=n}^{n+m-1} \tau_{i},
\end{aligned}
$$

where $R=\exp \left(\sum_{1=n}^{n+m-1} \sigma_{i}\right)$. That is,

$$
\left\|x_{n+m}-p\right\| \leq R\left[\left\|x_{n}-p\right\|+\sum_{i=n}^{n+m-1} \tau_{i}\right],
$$

for all $m, n \geq 1$, for all $p \in \mathscr{F}$ and for $R=\exp \left(\sum_{1=n}^{n+m-1} \sigma_{i}\right)>0$.

It follows from Lemma 2.3 that the limit $\lim _{n \rightarrow \infty}\left\|x_{n}-p\right\|$ exists.

By (3.10), we get

$$
d\left(x_{n+1}, \mathscr{F}\right) \leq\left(1+\sigma_{n}\right) d\left(x_{n}, \mathscr{F}\right)+\tau_{n}
$$

and it follows from condition

$$
\liminf _{n \rightarrow \infty} d\left(x_{n}, \mathscr{F}\right)=0
$$


and by Lemma 2.3 that

$$
\lim _{n \rightarrow \infty} d\left(x_{n}, \mathscr{F}\right)=0 .
$$

Next, we show that $\left\{x_{n}\right\}$ is a Cauchy sequence in X. Since $\lim _{n \rightarrow \infty} d\left(x_{n}, \mathscr{F}\right)=0$ and $\sum_{n=1}^{\infty} \tau_{n}<$ $\infty$, it follows that for any given $\epsilon>0$, there exists a positive integer $n_{0}$ such that for all $n \geq n_{0}$, $d\left(x_{n}, \mathscr{F}\right)<\frac{\epsilon}{6 R}$ and $\sum_{1=n}^{\infty} \tau_{i}<\frac{\epsilon}{3 R}$. In particular, $d\left(x_{n_{0}}, \mathscr{F}\right)<\frac{\epsilon}{6 R}$. Thus there must exist $p_{0} \in \mathscr{F}$ such that $\left\|x_{n_{0}}-p_{0}\right\|<\frac{\epsilon}{6 R}$.

Hence, for all $n \geq n_{0}$ and $m \geq 1$, we have

$$
\begin{aligned}
\left\|x_{n+m}-x_{n}\right\| & \leq\left\|x_{n+m}-p_{0}\right\|+\left\|x_{n}-p_{0}\right\| \\
& \leq R\left\|x_{n_{0}}-p_{0}\right\|+R\left(\sum_{1=n_{0}}^{n+m-1} \tau_{i}\right)+R\left\|x_{n_{0}}-p_{0}\right\|+R\left(\sum_{1=n_{0}}^{n-1} \tau_{i}\right) \\
& \leq 2 R\left\|x_{n_{0}}-p_{0}\right\|+2 R\left(\sum_{1=n_{0}}^{\infty} \tau_{i}\right) \leq 2 R \frac{\epsilon}{6 R}+2 R \frac{\epsilon}{3 R}=\epsilon
\end{aligned}
$$

which shows that $\left\{x_{n}\right\}$ is a Cauchy sequence in $\mathrm{X}$.

Thus, the completeness of $X$ implies that $\left\{x_{n}\right\}$ is convergent. Assume that $\left\{x_{n}\right\}$ converges to a common fixed point $p$ of $T_{1}, T_{2}, T_{3}$.

Then $p \in K$, because $K$ is closed subset of $X$. Therefore the set $\mathscr{F}=\bigcap_{i=1}^{3} F\left(T_{i}\right)$ is closed. $\lim _{n \rightarrow \infty} d\left(x_{n}, \mathscr{F}\right)=0$ gives that $d(p, \mathscr{F})=0$.

Thus $p \in \mathscr{F}$. This completes the proof.

In Theorem 3.1, if $T=T_{1}=T_{2}=T_{3}$, we obtain the following result:

Theorem 3.2. Let $X$ be a real Banach space and $K$ be a nonempty closed convex subset of $X$ and $T: K \rightarrow K$ be a generalized asymptotically quasi-nonexpansive mapping with respect to $\left\{r_{n}\right\}$ and $\left\{s_{n}\right\} \subset[0,1)$ and $\sum_{n=1}^{\infty} \frac{2 s_{n}+r_{n}}{1-s_{n}}<\infty$ with $\lim _{n \rightarrow \infty} r_{n}=0$ and $\lim _{n \rightarrow \infty} s_{n}=0$. Assume that $\sum_{n=1}^{\infty} \mu_{n 1}<$ $\infty, \sum_{n=1}^{\infty} \mu_{n 2}<\infty, \sum_{n=1}^{\infty} \mu_{n 3}<\infty$. Then the iterative sequence $\left\{x_{n}\right\}$ defined by (2.1) converges strongly a fixed point $p$ of $T$ if and only if $\liminf _{n \rightarrow \infty} d\left(x_{n}, p\right)=0$.

\section{References}

[1] M. K. Ghosh and L. Debnath, Convergence of Ishikawa iterates of quasi-nonexpansive mappings, J. Math. Anal. Appl., 207 (1997), 96-103.

[2] R. Glowinski, P. Le Tallec, Augmented Lagrangian and Operator-Splitting Methods in Nonlinear Mechanics, SIAM, Philadelphia, 1989.

[3] K. Goebel and W. A. Kirk, A fixed point theorem for asymptotically nonexpansive mappings, Proc. Amer. Math. Soc., 35 (1972), 171-174.

[4] H. Lan, Common fixed point iterative processes with errors for generalized asymptotically quasi-nonexpansive mappings, Computers and Math. with Applications 52 (2006), 1403-1412. 
[5] Q. H. Liu, Iterative sequences for asymptotically quasi-nonexpansive mappings, J. Math. Anal. Appl., 259 (2001), $1-7$.

[6] Q. H. Liu, Iterative sequences for asymptotically quasi-nonexpansive mappings with Error Member, J. Math. Anal. Appl., 259 (2001), 18-24.

[7] W. R. Mann, Mean value methods in iteration, Proc. Am. Math. Soc., 4 (1953), 506-510.

[8] I. Ishikawa, Fixed point by a new iteration method, Proc. Amer. Math. Soc., 44 (1974), 147-150.

[9] K. Nammanee and S. Suantai, The modified Noor iterations with errors for non-Lipschitzian mappings in Banach spaces, Appl. Math. Comput., 187 (2007), 669-679.

[10] J. Nantadilok, Three-step iteration schemes with errors for generalized asymptotically quasi-nonexpansive mappings, Thai J. Math., 6 (2008), 295-306.

[11] W. Nilsrakoo and S. Saejung, A new three-step fixed point iteration scheme for asymptotically nonexpansive mappings, Applied Mathematics and Computation, 181 (2006), 1026-1034.

[12] M. A. Noor, New approximation schemes for general variational inequalities, J. Math. Anal. Appl., 251 (2000), 217-229.

[13] W. V. Petryshyn and T. E. Williamson, Strong and weak convergence of the sequence of successive approximations for quasi-nonexpansive mappings, J. Math. Anal. Appl., 43 (1973), 459-497.

[14] G. S. Saluja and H. K. Nashine, Convergence theorems of fixed point for generalized asymptotically quasinonexpansive mappings in Banach spaces, Bulletin of Math. Anal. and Appl., 2(2010), 1-11.

[15] S. Suantai, Weak and strong convergence criteria of Noor iterations for asymptotically nonexpansive mappings, J. Math. Anal. Appl., 311 (2005), 506-517.

[16] K. K. Tan and H. K. Xu, Approximating fixed points of nonexpansive mappings by the Ishikawa iterative process, J. Math. Anal. Appl., 178 (1993), 301-308.

[17] B. L. Xu and M. A. Noor, Fixed point iterations for asymptotically nonexpansive mappings in Banach spaces, J. Math. Anal. Appl., 267 (2002), 444-453.

[18] L. Yang, X. Xie, S. Peng and G. Hu, Demiclosed principle and convergence for modified three step iterative process with errors of non-Lipschitzian mappings, J. Computational and Applied Maths., 234, (2010), 972984.

Department of Mathematics, Faculty of Science and Arts, Harran University, 63200, Sanliurfa, Turkey.

E-mail: temirseyit@harran.edu.tr

Department of Mathematics, Faculty of Sciences, Ataturk University, 25240, Erzurum, Turkey.

E-mail: hukmu@atauni.edu.tr 